\title{
Direct-to-chip liquid cooling for reducing power consumption in a subarctic supercomputer centre
}

\section{Seppo J. Ovaska*}

Department of Electrical Engineering and Automation,

Aalto University,

FI-02150 Espoo, Finland

Email: seppo.ovaska@aalto.fi

*Corresponding author

\section{Roy E. Dragseth and Svenn A. Hanssen}

Department of Information Technology,

UiT The Arctic University of Norway,

N-9037 Tromsø, Norway

Email: roy.dragseth@uit.no

Email: svenn@hanssen.priv.no

\begin{abstract}
Reduction of data centre power consumption is a timely challenge. Waste heat reuse is another focus area when developing energy efficient and sustainable data centres. And these two issues are interconnected through liquid cooling of server racks and/or direct-to-chip liquid cooling. Both of these solutions make it possible to transfer a significant proportion of the waste heat energy back to profitable use. Nevertheless, the heat reusing opportunity is not the only benefit direct-to-chip liquid cooling may offer. Another benefit is the notable reduction of power consumption related to cooling fans associated with server blades and rack-level cooling systems. To evaluate this benefit, we performed power consumption and performance measurements in a subarctic supercomputer centre hosting a cluster of 632 blade nodes. Our study concentrated on a 47-node subset that we analysed when the servers were executing the LINPACK benchmark. Our conclusion is that direct-to-chip liquid cooling can reduce the total power consumption, in this case, up to $14.4 \%$ depending on the inlet air temperature.
\end{abstract}

Keywords: data centre; direct-to-chip liquid cooling; power consumption; subarctic climate; supercomputer; waste heat reuse.

Reference to this paper should be made as follows: Ovaska, S.J., Dragseth, R.E. and Hanssen, S.A. (2016) 'Direct-to-chip liquid cooling for reducing power consumption in a subarctic supercomputer centre', Int. J. High Performance Computing and Networking, Vol. 9, No. 3, pp.242-249.

Biographical notes: Seppo J. Ovaska received his DSc degree from Tampere University of Technology in 1989. He is a Professor in the School of Electrical Engineering at Aalto University. In the academic year 2006 to 2007, he served as a Visiting Professor at Utah State University. His research expertise includes computational intelligence, green computing, and industrial electronics. He has published more than 100 articles in peer-reviewed journals, and he holds six US patents. Recently, he served as a Visiting Professor at UiT The Arctic University of Norway, where he worked on a new research initiative 'green computing in arctic environment'.

Roy E. Dragseth holds a Master's degree in Applied Mathematics from the University of Tromsø (UiT), and has worked with system administration and user support in the HPC Services at UiT for nearly 15 years. He has been the main system architect behind the last three generations of HPC systems deployed at UiT, and was deeply involved in the design of the current $0.5 \mathrm{MW}$ HPC data centre. Currently, he is working on the final design of the new $2 \mathrm{MW}$ data centre under construction.

Svenn A. Hanssen holds a Master's degree in Computer Science from the University of Tromsø (UiT), and has worked as an IT professional for more than 20 years. From 1996 to 2009, he was the Head of Systems Operations at UiT; and from 2009 to 2015, the Head of Research and Educational IT also at UiT. He has been responsible for establishing several generations of high performance computing systems and data centre infrastructures. From 2000 to 2004, he was a Project Manager of outreach and dissemination in the national HPC-program of Norway (NOTUR). He is currently the CEO at Serit IT Partner Tromsø AS. 


\section{Introduction}

Energy efficient and sustainable - or 'green' - data centre concepts are gaining increasing interest among data centre owners, operators, and consultants. But most organisations do not have the resources to deploy green-related technologies and techniques simply for environmental reasons - there has to be a business case or justification. This reality was emphasised by Schulz (2009) in his insightful book on green data centres. Therefore, the true motivation for green (or more precisely 'profitable-green') data centres is commonly related to achieving savings in total energy expenses while, at the same time, increasing the return on investment (ROI). The savings in operational expenses (OPEX) may come from two principal directions:

1 the electricity bill

2 the opportunity to utilise the waste heat energy for building and district heating.

In addition, the capital expenses (CAPEX) may be reduced by extending IT equipment refresh periods owing to the increased performance afforded by direct liquid cooling, or even by constructing a data centre in a cool subarctic environment.

Because the two OPEX-saving directions are usually interconnected through some liquid-cooling scheme of servers and IT accessories, we decided to study the effects of two liquid-cooling solutions to the total power consumption of a complete rack with 47 server nodes (each with two $2.8 \mathrm{GHz}$ Intel $^{\circledR}$ Xeon $^{\circledR}$ E5-2680v2 Ivy Bridge CPUs) and associated networking switch devices in active operation. This production-grade rack was a subset of the Stallo supercomputer cluster at UiT The Arctic University of Norway, which consisted of 328 HP SL230 Gen8 and 304 HP BL460c Gen8 blade servers, providing peak performance of 250 Teraflop/s. Our research is a part of the pre-evaluation phase of the greener Stallo 2 data centre with extensive waste heat reuse and $25 \%$ higher peak performance. The study was carried out at five inlet air temperatures between 20 and $30^{\circ} \mathrm{C}$ to gain understanding of the effects of higher-than-usual $\left(>25^{\circ} \mathrm{C}\right)$ computer room temperatures that could be feasible and cost effective when direct-to-chip liquid cooling is in use.

Although liquid cooling has been discussed in detail in numerous published articles during the past 20 years (Beaty and Smith, 2004), the topic is again timely, for instance, owing to the evolving viewpoint from effective cooling of CPU chips toward extensive waste heat reuse. And in this particular article, we address both of these complementary subjects. Nonetheless, there are understandable concerns on accidental leaking of coolants that have reduced the interest of conservative data centre operators to adopt liquid-cooling solutions (Beaty, 2004). But, on the other hand, there will always be less temperature fluctuation with direct-to-chip liquid cooling solutions than with traditional air cooling, which will undoubtedly have a positive effect to reliability. Our study began with a focused background survey on related topics to form a sound basis for the empirical phase.
This article is organised as follows. The background survey and different solutions for liquid cooling are first presented as a literature review in Section 2. Section 3 provides a motivating introduction to our measurement setups. Measurement results are given in Section 4 with a brief discussion. Section 5 closes this article, provides a summarising discussion on the evaluated liquid-cooling solutions, and suggests potential topics for future works.

\subsection{Contributions}

The main contributions of this empirical research experiment are outlined below, and they are discussed in detail in the following sections. These results are particularly useful for comparison purposes.

1 We measured and analysed the total power consumption of a subset of the Stallo HPC platform with two alternative liquid-cooling solutions implemented for a blade-server system that was originally designed to be fully air cooled. It was observed that significant power savings of $9.4 \%$ to $14.4 \%$ with practically equal computing performance were achieved when the hybrid of rack-level liquid cooling and direct-to-chip liquid cooling of CPUs was used instead of rack-level liquid cooling unaccompanied.

2 The computing performance of the 47-node Linux cluster was measured under the LINPACK benchmark execution, and the corresponding performance-per-watt metrics were calculated. The best performance/watt metric of our 47-node cluster was 1,068.8 MFLOPS/W with direct-to-chip liquid cooling, while it was $9.9 \%$ lower with pure rack-level liquid cooling.

\section{Literature review}

\subsection{Highlights from the background survey}

A comprehensive tutorial on the evolution of data centres was presented in Kant (2009), where the author discusses also the power and thermal management challenges that are at the core of green data centres. He points out that power efficiency without accounting for performance impact is not a useful metric for data centres. This is specifically true with supercomputing centres. Hence, we decided to compare also the performance-per-watt measures at different inlet air temperatures for the two liquid-cooling solutions under study. To keep the average 'performance' term nearly constant and unambiguous in comparisons and conclusions, all our server nodes were executing the same LINPACK benchmark throughout the measurement sessions.

Furthermore, Sun and Lee (2006) provide a pragmatic case study on data centres' energy performance. The authors discuss the indicators related to energy performance and define the term 'energy performance' as 'a comprehensive valuation of the energy use in data centre facilities, which involves energy consumption pattern, power load, power 
quality and reliability, systems' operational efficiency and associated cost'. Thus, our present study considers only one slice of that entity (i.e., the power load), which should be addressed as a whole when comparing alternative data centre concepts to each other. However, the studied data centres in Sun and Lee (2006) are located in a tropical environment, while our Stallo supercomputer is in the subarctic environment of Northern Norway with clearly different opportunities and constraints. The unique climate profile in this region means that there is virtually a constant demand for heating of buildings and other infrastructures, like pavements and runways. This is especially the case close to the ocean coast, where the annual temperature variation is much less than in the inland owing to the Gulf Stream. At the UiT campus in Tromsø (Latitude $69.6^{\circ} \mathrm{N}$ ), the outdoor temperature is $90 \%$ of time no more than $12^{\circ} \mathrm{C}$ (see Figure 1). In average, only 9 hours per year are warmer than $25^{\circ} \mathrm{C}$, and the annual average temperature is $4^{\circ} \mathrm{C}$. Hence, the forthcoming Stallo 2 installation in Tromsø which could be considered also as a 'heating plant' - will be a perfect test-bed for examining waste heat reuse during the whole year. And on exceptionally warm days, outdoor-air cooling of the supercomputer centre with dry-chillers is a practical backup solution. Furthermore, it should be emphasised that Stallo is located on the university campus with substantial heat reuse opportunities nearby.

Figure 1 Cumulative distribution of outdoor air temperatures in Tromsø during the lengthy period of last 20 years (see online version for colours)

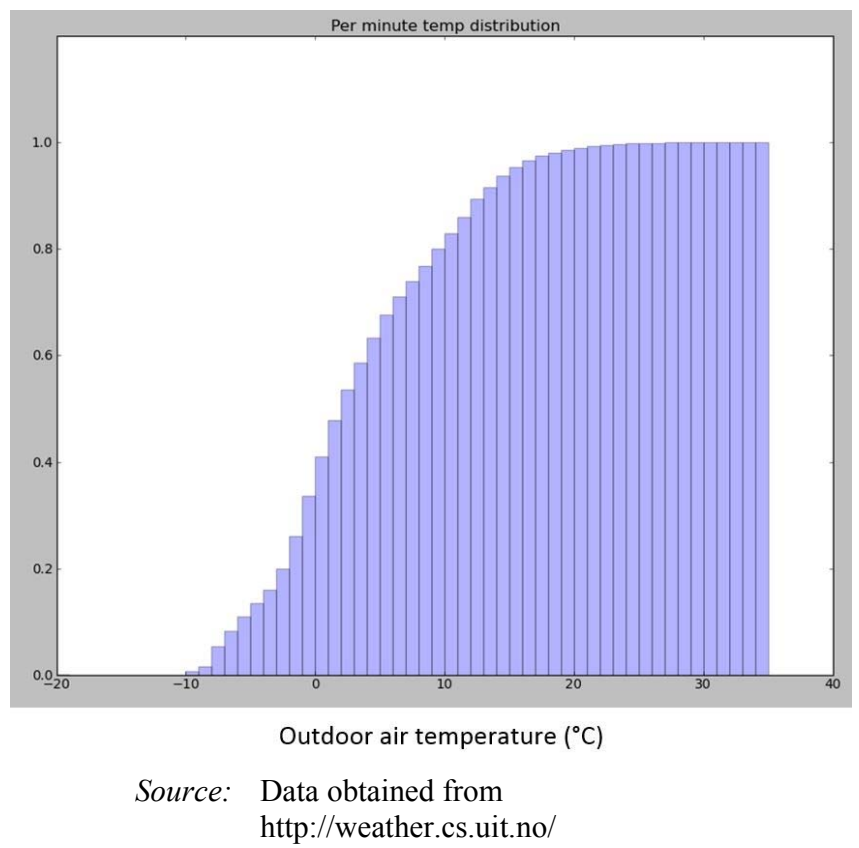

Ardito and Morisio (2014) take a broader point of view of green IT, and discuss generally applicable guidelines for saving energy without ruining the quality of service that the users experience. The authors state also that it would be necessary to standardise the data collection processes to make it possible to compare different studies on data centre energy efficiency objectively. This would be extremely useful for researchers and engineers working with similar challenges.

Finally, Bartolini et al. (2014) introduce Eurora, a kind of reference for green supercomputers, from the thermal and power viewpoints. Eurora was ranked first in The Green500 List (Feng and Cameron, 2007) in June 2013 with 3,208.83 MFLOPS/W. Eurora's outstanding energy efficiency is achieved by adopting a direct liquid-cooling solution for a heterogeneous system architecture. The role of direct liquid cooling is crucial in green data centres, particularly in HPC data centres where the computing load is scheduled close to $100 \%$ practically all of the time. Therefore, the other cooling solution we examine in this article is based on a hybrid of rack-level liquid cooling and direct-to-chip liquid cooling of CPUs, and it is compared to a pure rack-level liquid-cooling solution.

It should be reminded that although there are large data centres with power usage effectiveness (PUE) very near 1 that are not using direct liquid cooling, it does not necessarily mean that their overall energy consumption is 'relatively low' - it only tells that a very high proportion of consumed power is used by the computing equipment. By using direct liquid cooling, the total energy consumption could further be reduced, particularly in subarctic regions with a cold climate and significant heat reusing opportunities. Hence, how heat reuse should be accounted for is an important general subject to address. The data centre community has PUE, which is well understood and accepted, but when heat reuse is included, there is no widely accepted overall measure of goodness available - yet.

\subsection{Solutions for liquid cooling}

Cooling of data centres is a principal issue that must be handled effectively, because of high and continually increasing power densities of servers and racks. Currently, there may already be more than 100 times more energy per square metre in a data centre than in a typical office environment (Fakhim et al., 2011). The authors mentioned in their case study that 'as heat dissipation in [air-cooled] data centres rises by orders of magnitude, inefficiencies such as air recirculation causing hot spots and flow short-circuiting will have a significant impact on the thermal manageability and energy efficiency of the cooling infrastructure'. Thus, in green data centres aimed for high-performance computing, it is advantageous to use some form of direct liquid cooling instead of pure air cooling, since this would enhance the opportunity to reuse the waste heat more effectively and, simultaneously, increase the usage of the CPU turbo mode (Gepner et al., 2010). The thermal capacity of liquids is noticeably larger than that of air - air $1.00 \mathrm{~kJ} /(\mathrm{K} \cdot \mathrm{kg})$ and water $4.18 \mathrm{~kJ} /(\mathrm{K} \cdot \mathrm{kg})$, for instance - and after the heat energy has been transferred to water, it can be removed from the data centre efficiently (Coles et al., 2011). Moreover, for green data centres, water is usually the preferred liquid, because it is non-toxic, as well as more available and less expensive than other types of liquid. 
There are basically two extremes in liquid-cooling solutions, indirect rack-level (IRL) liquid cooling (i.e., in-row coolers, overhead coolers, and enclosed rack-level cooling) and direct component-level (DCL) liquid cooling; and as with any two boundaries, there are intermediate alternatives for hybrid solutions, too. These will be introduced shortly.

IRL liquid cooling may consist of a liquid-cooling package (LCP) for rack climate control (Rittal, 2013). Similar approaches are available from multiple vendors, such as the modular cooling system (MCS) discussed in HP (2008). The LCP has an inlet for cold water and outlet for warm water, and it contains a closed air circulation loop through the server rack and a heat exchanger. Fans are used for blowing the air effectively through servers, and both the air flow and water flow are regulated to maintain a desired inlet air temperature for the rack enclosure. From the component-cooling viewpoint, this solution corresponds to pure air cooling; but even with IRL liquid cooling, it is possible to reuse the heat energy from the warm-water outlet. Thus, this solution is often the first step toward a green data centre.

DCL liquid cooling, on the other hand, is targeted for cooling the CPUs and other such components, which are the main producers of waste heat, i.e., GPUs and memories. In these solutions, some coolant liquid is circulated with a very close proximity to the hot components. IBM BladeCenter QS22 is an example of factory-made DCL liquid cooling, and it is used in the Aquasar supercomputer prototype (Zimmermanna et al., 2012). In that specific case, the novel water-cooling solution is implemented using micro-channel heat sinks that bring water directly over the CPUs, and, hence, offer excellent heat transfer from the CPU chips to the water (Escher, 2009). In addition, it is possible to retrofit DCL liquid cooling on servers that were originally designed for air cooling. Such liquid-cooler assemblies are commercially available, and they are drop-in replacements for the CPU air heat sinks, and possibly for other heat sinks, too (HPCwire, 2014). This is illustrated in Figure 2, where we show side-by-side two server blades of the same type: one with the original air heat sinks and the other with retrofitted liquid coolers (the black hose with a blue ring brings in cool water and the one with a red ring brings out warm water) for the two 10-core CPUs. All the current DCL liquid-cooling solutions are meant to operate with a complementary air-cooling solution - internal or external because there does not exist any pure DCL liquid-cooling solution for large-scale data centres.

Hybrid IRL-DCL liquid cooling is an attractive solution for green HPC data centres. Externally, it has the benefits of full-blown liquid cooling, while internally it provides a competitive compromise and evolution step between pure air cooling and direct liquid cooling (Rubenstein et al., 2010; Zeighami et al., 2014). An interesting framework for comparing high performance computing technologies is proposed in Duran et al. (2014). Such a general framework could be used also in quantifying technology characteristics and assessing the most appropriate cooling technology for a particular HPC data centre.

Figure 2 A close-up of two HP SL230 Gen8 server blades; the left one with original air heat sinks and the right one with retrofitted liquid coolers (see online version for colours)

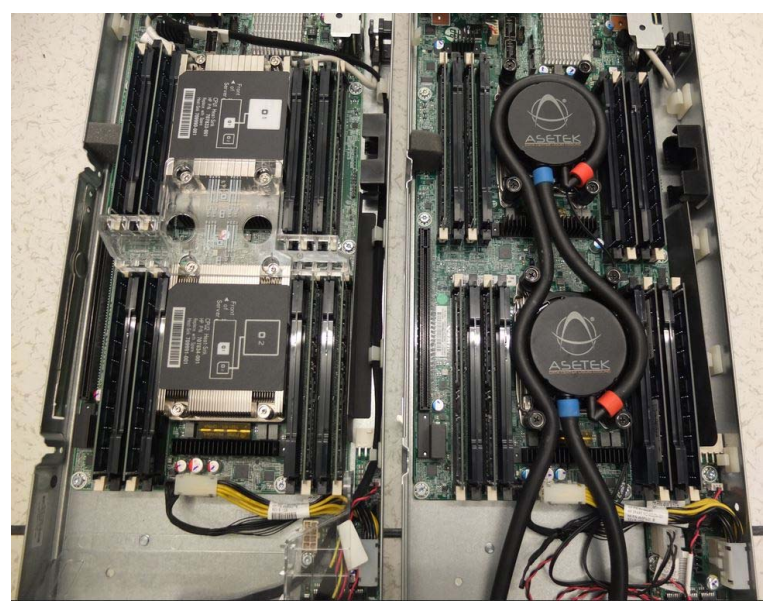

\section{Measurement setups}

A practical conclusion on the benefits of direct liquid cooling in data centres is available in Coles and Greenberg (2014), where the authors point out that the power consumption of the electronic equipment internal fans is significantly reduced when the data centre is equipped with a direct liquid-cooling system. To confirm and quantify this particular benefit in the Stallo supercomputer environment, we performed a series of power consumption and performance measurements that will be discussed below. The primary goal of these experiments was to find out how much the server and total (server and LCP together) power consumptions vary with different inlet air temperatures. In all our experiments, the server fans remained operational throughout the measurements, and the fans in the LCP were used to provide a neutral or positive delta pressure from the front to the rear of the server blades.

For the measurement sessions, we isolated a rack of 47 server nodes and communications switches from the regular production composition of Stallo. The peak performance of that 47-node HP SL230 Gen8 cluster rack is 21 Teraflop/s. To keep the average loading of nodes virtually constant, all the servers were executing the same HPL version of the LINPACK benchmark (Dongarra et al., 2003). High-performance LINPACK (HPL) is a software package that solves a dense system of linear equations, in 64-bit floating-point arithmetic on distributed-memory computers. The benchmark was run as a single-node benchmark utilising all CPU cores on the server and set up to restart immediately throughout the measurement sessions. With this computation-intensive benchmark, the CPU load on the nodes was $100 \%$ and the HPL benchmark achieved $85.7 \%$ to $86.7 \%$ of the theoretical peak performance (with 
turbo mode $\mathrm{ON}$ and hyper-threading $\mathrm{OFF}$ ), maintaining the average total power consumption relatively high, 17-20 kW, depending on the inlet air temperature. Although our experiments are based on a single server rack, the results can be scaled directly for multiple racks in our HPC environment. A visual performance monitoring toolkit for cluster environments was introduced in Li et al. (2009). Such a versatile toolkit would make the process of performance analysis more intuitive and convenient.

The rack under study was enclosed in a plywood cabinet (see Figure 3) to reduce air exchange with the computer room air (constant temperature $24^{\circ} \mathrm{C}$ ), while the temperature inside the cabinet was varied stepwise from 20 to $30^{\circ} \mathrm{C}$ with the step of $2.5^{\circ} \mathrm{C}$. Hence, the cabinet formed a controllable microclimate around the server rack.

Figure 3 The production-grade rack under study enclosed in a plywood cabinet to form a controllable microclimate around it (see online version for colours)

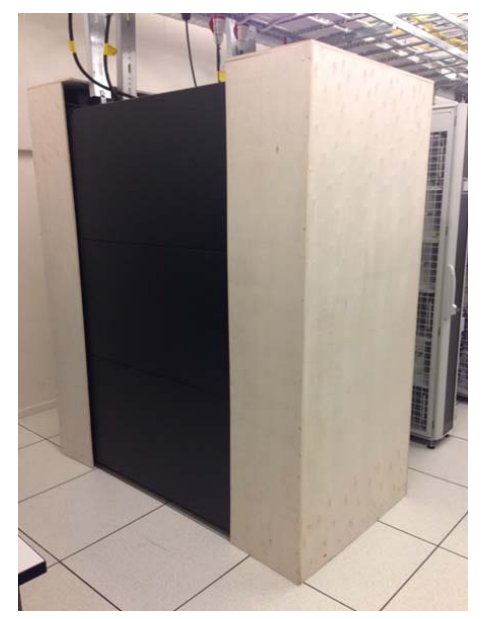

Our experiments were conducted under $100 \%$ computing load, since supercomputers are scheduled to run at very high loading all the time. Besides, it is well known that efficiency of cooling systems and servers is best at high loading. At lower loadings, cooling losses become greater and more cooling is wasted. Hence, it is likely that direct-to-chip liquid cooling would be less competitive at lower loadings, such as in cloud computing data centres with varying traffic (Zhanikeev, 2015) - but they are beyond this study.

\subsection{Session descriptions}

In the first measurement session, the cooling solution was of type IRL liquid cooling, and the inlet air temperature was the same as the regulated air temperature inside the plywood cabinet. It used the Rittal (2013) LCP for rack cooling. The fan and inlet liquid-flow controllers of the LCP were set to 'automatic' on temperatures from 20.0 to $25.0^{\circ} \mathrm{C}$, but at temperatures from 27.5 to $30.0^{\circ} \mathrm{C}$, the inlet liquid valve was controlled manually to reduce the potentially harmful fluctuations when increasing the air temperature; we just wanted to make sure that the inlet air temperature is not overshooting much above $30.0^{\circ} \mathrm{C}$, which could lead to overly high temperatures on the possible hot spots of server blades. How the servers react (power usage/turbo mode) during the experiments depends on the software load, environmental temperatures, and sealing of the plywood enclosure. However, the sealing between the cabinet and rack was apparently not perfect but was found adequate for our purpose.

Figure 4 Setups for the two measurement sessions with (a) IRL and (b) hybrid IRL-DCL cooling solutions (see online version for colours)

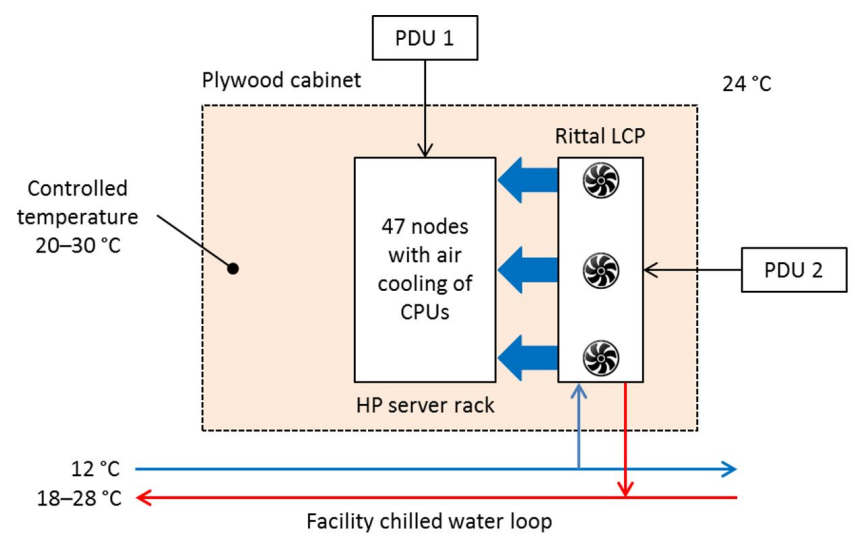

(a)

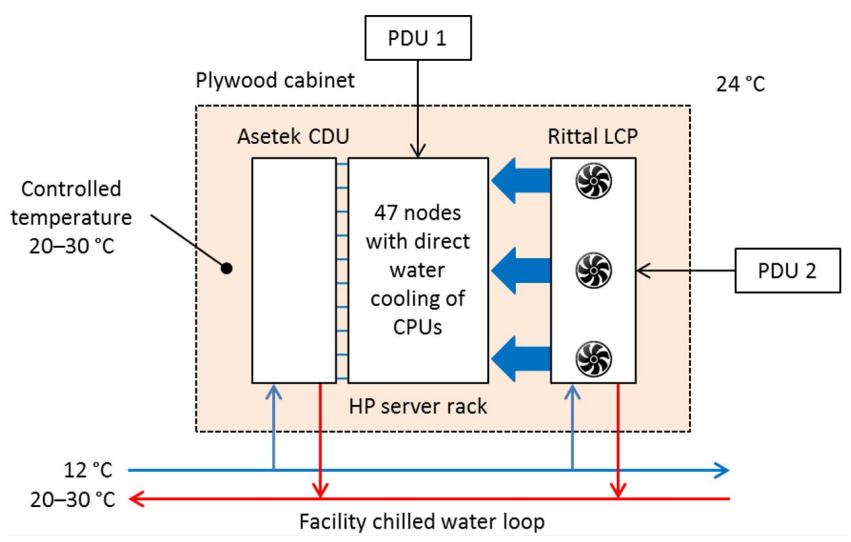

(b)

For the second measurement session, the same IRL liquid cooling solution was complemented with DCL liquid cooling that provided cooling for CPUs only. That was an Asetek CDU retrofit direct-to-chip liquid cooling system shown on right in Figure 2 (HPCwire, 2014). Note: the vendor names of key components are mentioned here solely to make this article worthwhile for practitioners.

In both of the sessions, the inlet water temperature was fixed $12^{\circ} \mathrm{C}$, while the outlet water temperature had an approximative value of $18-28^{\circ} \mathrm{C}$ and $20-30^{\circ} \mathrm{C}$ in the first and second measurement sessions, respectively. The notably cold inlet water was coming from a general-purpose chiller that is used not only for cooling the data centre but also for cooling other university facilities. Both of the measurement setups are sketched in Figure 4. For effective reusing of waste heat under these conditions in radiator heating, a heat pump (typical efficiency $75 \%$ ) is needed to increase the outlet water temperature above $45^{\circ} \mathrm{C}$. However, even the 
$\sim 30^{\circ} \mathrm{C}$ water temperature would be adequate for floor heating and pavement heating purposes, for example.

It should be noted that these experiments do not take into consideration the chiller power consumption. All the experiments assume that cold water is available anyway from the large general-purpose chiller. However, the IRL (1st session) and hybrid IRL-DCL (2nd session) cooling solutions were connected to exactly the same chilled water supply as shown in Figure 4. In the future, when the Stallo 2 environment is complete and that chiller is no more in use, we are going to record the total power consumption periodically throughout the year.

\section{Power consumption and performance measurements}

The average power consumptions of servers and the LCP were measured from the outputs of two power distribution units, PDU 1 and PDU 2 in Figure 4, over an average period of 22 minutes when the inlet air temperature had no more than marginal variation around its set point. In addition, the corresponding average performance metric of each server node was recorded for inlet air temperatures 20,25, and $30^{\circ} \mathrm{C}$. These measurements were carried out in the summer of 2014.

Table 1 Average power consumptions with air (A) and liquid (L) CPU cooling at different inlet air temperatures

\begin{tabular}{cccccc}
\hline$A / L-{ }^{\circ} C$ & $\begin{array}{c}\text { Servers } \\
k W\end{array}$ & $\begin{array}{c}L C P \\
k W\end{array}$ & $\begin{array}{c}\text { Total } \\
k W\end{array}$ & $\begin{array}{c}\text { Reduction } \\
k W\end{array}$ & $\begin{array}{c}\text { Reduction } \\
\%\end{array}$ \\
\hline $\mathrm{A}-20.0$ & 17.935 & 0.877 & 18.812 & - & - \\
$\mathrm{L}-20.0$ & 16.752 & 0.288 & 17.040 & 1.772 & 9.4 \\
$\mathrm{~A}-22.5$ & 18.223 & 0.886 & 19.109 & - & - \\
$\mathrm{L}-22.5$ & 16.794 & 0.257 & 17.051 & 2.058 & 10.8 \\
$\mathrm{~A}-25.0$ & 18.616 & 0.907 & 19.523 & - & - \\
$\mathrm{L}-25.0$ & 16.891 & 0.246 & 17.137 & 2.386 & 12.2 \\
$\mathrm{~A}-27.5$ & 18.959 & 0.918 & 19.877 & - & - \\
$\mathrm{L}-27.5$ & 16.991 & 0.230 & 17.221 & 2.656 & 13.4 \\
$\mathrm{~A}-30.0$ & 19.226 & 0.915 & 20.141 & - & - \\
$\mathrm{L}-30.0$ & 17.127 & 0.105 & 17.232 & 2.909 & 14.4 \\
\hline
\end{tabular}

Table 1 gives the average power consumptions in kilowatts for both of the measurement sessions described in Section 3.1. It provides also the absolute $(\mathrm{kW})$ and relative $(\%)$ reductions of total average power when DCL is used. In Table 2, the corresponding median and mean node performances (Gigaflop/s), as well as the standard deviation (Std.) are given. Finally, Table 3 contains the cluster performance per watt (MFLOPS/W) that is computed from the data of Tables 1 and 2. The 'MFLOPS/W' metric is used here, because it is used also when compiling The Green500 List (Feng and Cameron, 2007). In addition, virtual Green500 ranks are given to indicate the relative greenness of our computing platform compared to other energy-efficient supercomputers around the world. The temperature values given in Tables 1 to 3 are the set point values, which correspond very well with the true average temperatures over the measuring period (virtual 'steady states').

Table 2 Server node (10+10 CPU cores) performance with air (A) and liquid (L) CPU cooling at different inlet air temperatures

\begin{tabular}{cccc}
\hline A/L- ${ }^{\circ} \mathrm{C}$ & $\begin{array}{c}\text { Median } \\
\text { Gigaflop/s }\end{array}$ & $\begin{array}{c}\text { Mean } \\
\text { Gigaflop/s }\end{array}$ & $\begin{array}{c}\text { Std. } \\
\text { Gigaflop/s }\end{array}$ \\
\hline A-20.0 & 386.2 & 385.6 & 6.3 \\
L-20.0 & 389.2 & 387.5 & 6.0 \\
A-25.0 & 383.9 & 384.1 & 6.8 \\
L-25.0 & 389.4 & 388.1 & 5.8 \\
A-30.0 & 384.8 & 384.2 & 6.7 \\
L-30.0 & 389.1 & 388.4 & 3.8 \\
\hline
\end{tabular}

Table 3 Cluster performance per watt with air (A) and liquid (L) CPU cooling at different inlet air temperatures

\begin{tabular}{ccc}
\hline$A / L-{ }^{\circ} C$ & $\begin{array}{c}47 \text { server nodes } \\
\text { MFLOPS } / W\end{array}$ & $\begin{array}{r}\text { 'Green500 rank' virtual } \\
\text { November } 2014^{1}\end{array}$ \\
\hline A-20.0 & 963.4 & 108 th \\
L-20.0 & $1,068.8$ & 98 th \\
A-25.0 & 924.7 & $121 \mathrm{st}$ \\
L-25.0 & $1,064.4$ & 98 th \\
A-30.0 & 896.5 & 136th \\
L-30.0 & $1,059.4$ & 98 th \\
\hline
\end{tabular}

Note: ${ }^{1}$ This virtual 'Green500 rank' is provided solely for giving an idea of the relative greenness of the cluster under study; $\mathrm{http} / / / \mathrm{www}$. green500.org/news/green500-listnovember-2014.

\section{Discussion}

\subsection{Power consumption}

Power consumption and performance measurements were carried out with a cluster of 47 server nodes under varied temperature conditions. The increase in total average power consumption when the inlet air temperature was stepped from 20 to $30^{\circ} \mathrm{C}$ was considerably higher with air-cooled CPUs (7.1\%) than with direct-to-chip liquid cooling (1.1\%); see Table 1 . This is because the system fans have to work much harder to keep the servers properly cooled at higher temperatures. In the case of direct-to-chip liquid cooling, the role of system fans is not as critical, because the CPUs the main producers of waste heat - are cooled effectively by a retrofitted liquid-cooler. Besides, the total average power consumption was $9.4 \%$ to $14.4 \%$ lower with direct-to-chip liquid cooling than with air-cooled CPUs. This result confirms and quantifies the observation of Coles and Greenberg (2014) that was mentioned in Section 3. The contribution of LCP rack cooling to the total average power consumption is $4.5 \%$ to $4.7 \%$ with air-cooled CPUs, but only $0.6 \%$ to $1.7 \%$ with direct-to-chip liquid cooling, as can 
be calculated from Table 1 . Hence, $95.3 \%$ to $99.4 \%$ of the consumed total power is used by the server computers, network switches, and their associated system fans (including losses of local power converters), as detailed in Figure 5. From Figure 5, we can observe that the red curve is consistently more than $3 \%$ units above the blue one. The power consumption of the network switches was not measured in the tests, but can be considered to be equal and constant in both sessions. Since the benchmarks ran solely within each node and thus did not cause any network communications one can reasonably assume that the network equipment ran at idle power throughout the tests. Furthermore, the power consumption of the Asetek CDU is negligible when compared to the other power levels in these experiments.

Figure 5 Relative usage of the consumed total power (\%) by the server nodes and network switches as a function of inlet air temperature $\left({ }^{\circ} \mathrm{C}\right)$ for air-cooled (diamonds) and liquid-cooled (squares) CPUs (see online version for colours)

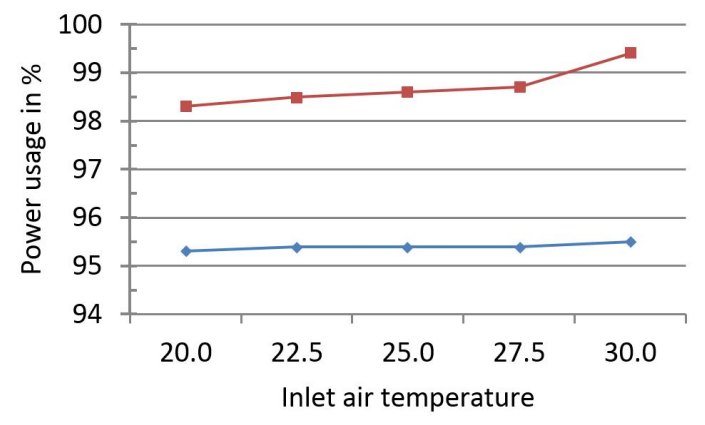

\subsection{Performance and greenness}

As can be seen from Table 2, the median performances with liquid-cooled CPUs remain practically constant at all inlet air temperatures, and the median performances with air-cooled CPUs vary just marginally. In general, median is an appropriate measure for this kind of comparison, because it effectively filters out the influence of possible outliers. All the 47 nodes behaved very consistently at all temperatures; the standard deviation of performance being only $1.0 \%$ to $1.8 \%$ of the corresponding mean value. Besides, it is observable from the results of Table 2 that the liquid-cooled CPUs provided slightly higher performance than the air-cooled ones; the performance measures of air-cooled CPUs were $0.5 \%$ to $1.1 \%$ lower. These differences are likely related to the fact that when direct-to-chip liquid cooling is used, the CPU chips stay cooler (in average), and, hence, the nodes are able to spend more time in the temporary turbo mode where the CPU clock rate is up to $10 \%$ higher. Nonetheless, the cooling solution does not have a significant effect on computing performance in our experiments.

After measuring the average power consumption and performance of the 47-node cluster at different inlet air temperatures, we computed the corresponding MFLOPS/W measures and related them to those of The Green500 List of November 2014 (see Table 3). Now, there is a considerable difference between the CPU cooling schemes: direct-to-chip liquid cooling clearly outperforms the air-cooling solution. The performance per watt with liquid cooling is $10.9 \%$ to $18.2 \%$ higher, and it would place the liquid-cooled cluster to the 98th rank on the semi-annually updated list. Moreover, it is notable that this rank does not change as the inlet air temperature steps from 20 to $30^{\circ} \mathrm{C}$. The situation is quite different with air-cooled CPUs: the virtual Green500 rank is comparatively dependent on the inlet air temperature, and it varies from the 108th to the 136th.

\subsection{Future works}

In the future, if the computer room temperature could be raised from the present $24^{\circ} \mathrm{C}$ to, say, $27^{\circ} \mathrm{C}$, we could expect around $13 \%$ reduction in total power consumption of servers and the LCP with direct-to-chip liquid cooling of CPUs. Such a moderate temperature increase could be realisable in the new $250 \mathrm{~m}^{2}$ and up to $2 \mathrm{MW}$ Stallo 2 data centre with extensive reuse of waste heat. However, before we can raise confidently and objectively the computer room temperature, we have to analyse the power consumption and heat capture issues with different inlet and outlet water temperatures, too, and find optimal settings for various temperature and flow parameters - without forgetting the reliability of the computer system and its components that may degrade in higher ambient temperatures. These will be central issues for our future studies, which could be carried out in the completed Stallo 2 HPC environment at the end of 2015.

To conclude, the direct-to-chip liquid cooling solution, studied in this article, is meant for providing an effective foundation for reusing waste heat, already in the near future. In fact, waste heat recovery is a key issue in the mission of UiT to become a leader in Green High Performance Computing.

\section{Acknowledgements}

The authors would like to thank S. Trædal-Henden for his assistance with the measurement setups. Moreover, the authors are grateful to the anonymous reviewers and the editor for their insightful comments and suggestions, which improved the manuscript significantly. The corresponding author acknowledges partial financial support from the Aalto ELEC Energy Programme.

\section{References}

Ardito, L. and Morisio, M. (2014) 'Green IT - available data and guidelines for reducing energy consumption in IT systems', Sustainable Computing: Informatics and Systems, Vol. 4, No. 1, pp.24-32.

Bartolini, A., Cacciari, M., Cavazzoni, C., Tecchiolli, G. and Benini, L. (2014) 'Unveiling Eurora - thermal and power characterization of the most energy-efficient supercomputer in the world', in Proceedings of the Conference on Design, Automation and Test in Europe, Dresden, Germany. 
Beaty, D. (2004) 'Liquid cooling of electronic equipment: friend or foe', in Proceedings of the ASHRAE Annual Meeting, Nashville, TN, USA.

Beaty, D. and Smith, R. (2004) 'Back to the future - liquid cooling: data center considerations', ASHRAE Journal, Vol. 46, No. 12, pp.42-46.

Coles, H. and Greenberg, S. (2014) Direct Liquid Cooling for Electronic Equipment, Technical Report LBNL-6641E, Lawrence Berkeley National Laboratory, Berkeley, CA, USA.

Coles, H., Ellsworth, M. and Martinez, D.J. (2011) 'Hot' for warm water cooling', in Proceedings of the International Conference for High Performance Computing, Networking, Storage and Analysis, Seattle, WA, USA.

Dongarra, J., Luszczek, P. and Petitet, A. (2003) 'The LINPACK benchmark: past, present, and future', Concurrency: Practice and Experience, Vol. 15, No. 9, pp.803-820.

Duran, R.E., Chen, D., Saraswat, R. and Hallmark, A. (2014) 'A framework for comparing high performance computing technologies', Int. J. Computational Science and Engineering, Vol. 9, Nos. 1/2, pp.119-129.

Escher, W. (2009) Ultra Thin High Efficiency Heat Sinks with Water or Nanofluid for Electronics, Doctoral Dissertation, Eidgenössische Technische Hochschule ETH Zürich, Zürich, Switzerland.

Fakhim, B., Behnia, M., Armfield, S.W. and Srinarayana, N. (2011) 'Cooling solutions in an operational data centre: a case study', Applied Thermal Engineering, Vol. 31, Nos. 14/15, pp.2279-2291.

Feng, W-C. and Cameron, K.W. (2007) 'The green500 list: encouraging sustainable supercomputing', Computer, Vol. 40, No. 12 , pp.50-55.

Gepner, P., Kowalik, M.F., Fraser, D.L. and Wackowski, K. (2010) 'Early performance evaluation of new six-core Intel ${ }^{\mathbb{B}}$ Xeon $^{\circledR} 5600$ family processors for HPC', in Proceedings of the 9th International Symposium on Parallel and Distributed Computing, Istanbul, Turkey, pp.117-124.

HP (2008) HP Modular Cooling System Generation 2 Water Cooling Technology, Online Document [online] http://h20566.www2.hpe.com/hpsc/doc/public/display?docId $=$ emr_na-c01490034\&lang $=$ en-us\&cc $=$ us (accessed 14 August 2015)
HPCwire (2014) UiT Recycles Supercomputing Power with Asetek's RackCDU TM, Online Document [online] http://www.hpcwire.com/2014/06/23/uit-recyclessupercomputing-power-aseteks-rackcdu/ (accessed 14 August 2015).

Kant, K. (2009) 'Data center evolution: a tutorial on state of the art, issues, and challenges', Computer Networks, Vol. 53, No. 17, pp.2939-2965.

Li, K-C., Hsu, C-H., Wen, C-H., Wang, H-H. and Yang, C-T. (2009) 'A dynamic and scalable performance monitoring toolkit for cluster and grid environments', Int. J. High Performance Computing and Networking, Vol. 6, No. 2, pp.91-99.

Rittal (2013) Liquid Cooling Package LCP Cooling Systems, Online Document [online] http://www.rittal.com/imf/none/5_916/ (accessed 14 August 2015).

Rubenstein, B.A., Zeighami, R., Lankston, R. and Peterson, E. (2010) 'Hybrid cooled data center using above ambient liquid cooling', in Proceedings of the 12th IEEE Intersociety Conference on Thermal and Thermomechanical Phenomena in Electronic Systems, Las Vegas, NV, USA.

Schulz, G. (2009) The Green and Virtual Data Center, CRC Press, Boca Raton, FL, USA.

Sun, H.S. and Lee, S.E. (2006) 'Case study of data centers' energy performance', Energy and Buildings, Vol. 38, No. 5, pp.522-533.

Zeighami, R., Coles, H., Saunders, W.A. and Branton, S. (2014) 'Thermal performance modeling of hybrid liquid-air cooled servers', in Proceedings of the 14th IEEE Intersociety Conference on Thermal and Thermomechanical Phenomena in Electronic Systems, Orlando, FL, USA.

Zhanikeev, M. (2015) 'A holistic community-based architecture for measuring end-to-end QoS at data centres', Int. J. Computational Science and Engineering, Vol. 10, No. 3, pp.315-324.

Zimmermanna, S., Meijerb, I., Tiwaria, M.K., Paredesb, S., Michelb, B. and Poulikakosa, D. (2012) 'Aquasar: a hot water cooled data center with direct energy reuse', Energy, Vol. 43, No. 1, pp.237-245. 